(Aus dem physiologischen Laboratorium in Bonn.)

\title{
Lässt sich durch mechanische Auslese des Fettes Fleisch von bestimmtem Nährwerth gewinnen?
}

\author{
Von
}

Heinrich Steil.

Bei Untersuchungen über den Stoffwechsel des Menschen und der Thiere liegt eine grosse Schwierigkeit in der Beurtheilung des Nährwerthes des Fleisches, weil dieses einen so wechselnden Fettgehalt nicht bloss von einem Thiere zum andern, sondern sogar von einem Muskel zum andern bei demselben Thiere darbietet. Wir finden Kubfleisch, welches noch nicht $1 \%$, anderes, welches mehr als $15 \%$ Fett enthält. (F. K ön i g II 191.) Es kann also der Fettgehalt des Fleisehes an Gewicht den Eiweissgehalt erreichen, ja iibertreffen, so dass das in diesem Fleisch enthaltene Fett an Näbrwerth sämmtliche anderen Bestandtheile des Fleisches mit Einschluss des Eiweisses bei Weitem übertrifft. In anderen Fällen kann der Fettgehalt des Fleisches auch so sehr, bis etwa $1 / 2 \%$, sinken, dass. dann derselbe nur noch einen kleinen Bruchtheil des Näbrgehalts ausmacht. Da man dem Fleische die Fetteinlagerung ansieht, die sich als eine weisse Marmorirung des sonst rothen Querschnittes darstellt, so sollte man meinen, dass sich der Fettgehalt eines gegebenen Fleisches annähernd schätzen liesse. Die in dem hiesigen Laboratorium ausgeführten zahlreichen Fettanalysen von Fleisch verschiedenster Herkunft haben aber ergeben, dass eine Schätzung. in hohem Grade unsicher ist. Ja es kommt Fleisch vor, welches auf dem Querschnitt rein roth ist, im Allgemeinen keine Fetteinlagerung dem unbewaffneten Auge darbietet und trotzdem mehrere Prozent Fett enthält. Prof. P. A rgut in s k y hat besonders auffallend diese Thatsache am Musculus pectoralis der Taube im hiesigen Laboratorium feststellen können. Da also im allgemeinen 
die Fettanalyse des Fleisches nicht umgangen werden kann, hentigen Tages aber die frülern quantitativen Methoden zur Bestimmung des Fettes nicht mehr als zuverlässig anerkannt werden können, so war eine erneute Prïfung der Frage geboten. Der Aufforderung des Herpn Professor Dr. Pfliig e r folgend, habe ich dieselbe unter seiner Leitung, so weit es meine beschränkte Zeit gestattete, zu bearbeiten mich entschlossen.

C. Voit ist der Erste, welcher zu Fütterungsversnchen durch mechanisehe Auslese des Fettes sich Fleisch von besti m m tem niederen Fettgehalte zu verschaffen suchte.

Die Literatur ergibt übrigens bereits, dass die bei der Auslese aufgewendete Sorgfalt, sowie die verschiedene Natur des Fleisches einen erheblichen Einfluss auf den Fettgebalt des geputzten Fleisches ausüben.

Als Beleg mögen folgende Analysen einiger Forscher angefiibrt werden, welche sämmtlich behanpten, das untersuchte Fleisch vor der Analyse von allem makroskopisch sichtbaren Fett sorgfältigst befreit zu haben.

Den niedrigsten Werth für den Fettgehalt gibt Gro a ve n ${ }^{1}$ ) an, der bei einem magern Bullen 0,61\% gefunden hat. Eine bei derselben Gelegenheit von ihm mitgetheilte Analyse des Fleisches eines andern, ebenfalls magern Thieres lieferte das Resultat von $1,83 \%$. Le b mann ${ }^{2}$ ) fand bei der Untersuchung verschiedener Stücke im Maximum 2,3\%, im Minimum 1,5\%. Petersen ${ }^{3}$ ) untersuchte zuerst das Fleisch von verschiedenen Stellen desselben Thieres. Er bestimmte das Fett im Vorder- und Hintertheil, woraus sich ergab:

$\begin{array}{cc}\text { Vorderschenkel } & \text { Hinterschenkel } \\ \text { Rind A } 0,76 \% & 3,1 \% \\ \text { B } 0,86 \% & 3,4 \%\end{array}$

Eine sich iiber noch mehr Theile desselben Thieres erstreckende Analyse theilen Leyder und $\mathrm{Pyr}^{4}$ ) mit. Dieselben unter-

1) Grouven, Physiologisch-chemische Fütterungsversuche. 1864. S. 84 .

2) Citirt bei Grouven a. a. O. S. 84 .

3) P etersen, Die Schwankungen im Wasser-, Fett- und Stickstoffgehalt des Fleisches. Zeitschr. für Biol. VII. Bd. S. 173.

4) Leyden und Py ro, La viande de boeuf et la viande de cheval 
suchten die Hals-, Bein-, Bauch- und Lendenmusculatur und fanden für:

$$
\begin{array}{ll}
\text { Hals } & 1,28 \% \\
\text { Schwanzstïck } & 0,92 \% \\
\text { Konterfilet } & 0,78 \% \\
\text { Filet } & 2,62 "
\end{array}
$$

Es ist klar, dass die von verschiedenen Forschern erhaltenen Werthe an Fett von der Leistungsfähigkeit und Zuverlässigkeit der zur quantitativen Fetthestimmung selbst angewandten analytischen Methode abhängig sind. Meist fehlen Angaben über die angewandte Methode der Fettbestimmung im Fleische, und grade dieses bietet ganz besondere Schwierigkeiten für die Beurtheilung. Meist wird nur erwähnt, dass zur Extraction des Fettes wasserfreier Aether verwandt worden sei.

Eines andern Extractionsmittels bediente sich $M$ è n $\mathrm{e}^{1}$ ), welcher in einer umfassenden Untersuchung über die Zusammensetzung des Fleisches des Pariser Seblachtviehes das Fett dadurch bestimmte, dass er aus dem feuchten Fleisch dasselbe mit Schwefelkohlenstoff entfernte. Die Ergebnisse seiner Arbeit sind aber von Bertram und S c häf e $r^{2}$ ) einer vernichtenden Kritik unterzogen worden, indem sie aus der Berechnung der von ihm gewonnenen Zahlen der einzelnen Bestandtheile und der Elementar-Analyse aufeinander den Beweis für die Unmüglichkeit seiner Zahlen erbrachten. M è n e's Verfahren kann also für analytisehe Zwecke nicht mehr in Betracht kommen.

Salkowski3) empfieblt ein Verfahren, das darin besteht, dass das Fleisch getrocknet, gepulvert und dann im Sox blet'schen Apparate drei Stunden mit heissem Aether extrahirt wird. Die auf diese Weise für den Fettgehalt gefundenen Werthe sind aber,

recherche sur leur composition leur valeur et leur prix commercial. Journal de médicine de Bruxelles 1874. S. 473.

1) Mène $\mathrm{Ch}$., Analyses de divers morceaux de viande de veau, de mouton, et de porc vendus courrament à la Halle de Paris en 1873 et 1874 . Comp. rend. Tom. 79. p. 529.

2) J. Bertram und M. Schäfer, Mène's Analysen des Pariser Schlachtfleisches und ihr Werth. Zeitschr. f. Biol. Bd. XII. S. 558. Chemie.

3) Salkowski, Prakticum der physiologischen und pathologischen 
wie P fl li ge $\mathrm{r}^{1}$ ) bei der Bestimmung des Fettes im Pferdefleisch nachgewiesen hat, zu niedrig. Wäbrend am ersten Tage aus der aufs feinste gepulverten Trockensubstanz von 3 bis $5 \mathrm{gr}$ eine Menge von $120-150 \mathrm{mg}$ Aetherextract gewonnen wurde, erhielt man am zweiten Tage noch 20, am dritten Tage noch 10 und in den folgenden 14 Tagen noch Quanta von 1 bis $5 \mathrm{mg}$. Den Grund für diese Thatsache fand Pf I ï ge r mit Recht darin, dass der Aether nur langsam in die Substanz eindringen und das Fett auflösen kann. Es kommt bei dem Ausziehen des Fettes mit Aether offenbar hauptsächlich darauf an, dass man dem Aether Gelegenheit bietet, wirklich bis zu dem Fette, welches er auflösen soll, hinzugelangen. Aus diesem Grunde hat P fl ü ger a. a. O. eine neue Methode angegeben, welche darin besteht, dass die thieriscien Gewebe, in denen der Fettgehalt bestimmt werden soll, zuerst in 5,10 bis 20 prozentiger Citronensäurelösung anf siedendem Wasserbad aufgelöst werden. Das Fett wird nach dem Erkalten abgeschöpft und die saure Lösung mit Aether ausgeschiittelt, in dem die Citronensäure nur sehr wenig löslich ist; die Aetherauszüge werden darnach mit Wasser ausgeschüttelt und darauf der Aether verdunstet. Die Auflösung mit heisser Citronensäure gelingt nicht bloss bei Fleisch, sondern auch bei den meisten andern Geweben, sogar bei Knochen.

A rguti n sky ${ }^{2}$ ) wandte den Soxhlet'schen Extractor in der Weise an, dass er oft eine Woche lang auszog und das Fleiseh nach ein- bis zweitägiger Behandlung pulverte, ehe der Prozess weiter fortgesetzt wurde. Zuweilen pulverte er noch ein zweites Mal. Die Resultate hierbei waren die, dass er die Hanptmenge am ersten Tage erhielt, am zweiten nur noch $1 / 20$ bis $1 / 30$ tel der erstern und am Schlusse stets nur noch ganz geringe Mengen. Hiermit- hielt er die Extraction für erschöpft. In der That sollte man glauben, dass, nachdem das Fleisch in fein gepulvertem Zustande 8. Tage lang ununterbrochen von siedend warmem fettfreien Aether (wie es im S o x b l e t'schen Apparate ja der Fall ist) durchströmt war, nun auch die letzten Spuren von Fett aus demselben ausgewaschen sein miissten.

1) Pflüger's Archiv Bd. 51. S. 277.

2) Argutinsky, P., Ueber die elementare Zusammensetzung des Ochsenfleisches. Pflüger's Archiv. Bd. 55. 
Indessen wies Dr. D or n e y e r, der chemische Assistent des physiologischen Instituts, in einer ìm Bonner Laboratorium auf Veranlassung des Herro Prof. Pfl ü ge r ausgefülrten und erst durch eine vorlänfige Mittlueilung (siehe Seite 341) veröffentlichten Untersuchung nach, dass aus dem nach dieser Art behandelten Fleisch bei immer weiterem Pulverisiren und Ausziehen mit Aether sich noch bedeutende Mengen Fett erhalten lassen. Es zeigt sich also, dass, je weiter man die mechanische Zerkleinerung des Fleisches fortsetzte, um so mehr Fett ausgezogen wurde, ohne dass aber eine vollständige Erschöpfung anf diesem Wege selbst bei Monate lang fortgesetzter Behandlung zu erreichen war. Nach jedem neuen Pulverisiren' kam zu der schon ausgezogenen Fettmenge ein neuer Summand hinzu; diese Summanden wurden zwar immer kleiner, lieferten aber trotztem fortgesetzt Beträge, deren Summe nicht zu vernachlässigen war. Da aus diesen Erfahrungen hervorging, dass durch das einfache mechanische Pulverisiren das Fett dem Eindringen des Aethers nicht genïgend zugänglich gemacht werden konnte, soweit man auch das Pulverisiren treiben mochte, so kam $P$ flüge $r$ auf den Gedanken, das Fleisch dadurch der Fettextraction durch Aether zugänglich zu machen, dass dasselbe vorher durch die Magenverdauung aufgelöst würde. Dor meyer wandte dieses Verfahren auf Fleischpulver an, das bereits mehrere hundert Stunden im So $\mathrm{x}$ b 1 e t'schen Apparate unter fortgesetzt wiederholtem Pulverisiren behandelt worden war, und fand in demselben noch bis $z \mathfrak{z}$ $0,75 \%$ Fett im Durchschnitt.

Mit dieser Methode, erst das durch Verdauung aufgelöste Fleisch mit Aether auszuschiitteln, war endlich ein Verfahren gefunden, mittelst dessen es gelingt, den ganzen Aetherextract des Fleisches olne Rest zu erhalten, wie dies D o r m e y e r näher begrtinden wird. Da nach den Ergebnissen der D o r m e y e r'schen Versuche klar war, dass die Werthe aller bisherigen Fettbestimmungen zu niedrig sind, so habe ich diese Methode anf das magere Kuhfleisch angewandt, um zuverlässige Werthe über den Fettgehalt desselben zu erhalten. Diese Frage ist von der grössten Wichtigkeit sowohl für die Beurtheilung der ältern Stoffwechseluntersuchungen, bei denen, wie bei den Vo i t'schen, mageres Rindfleisch zur Fütterung verwandt wurde, als auch für das Urtheil darïber, ob man bei späteren Stoffwechselversuchen berechtigt ist, für mage- 
res und von sichtbarem Fett möglichst befreites Fleisch - ohne Analyse - einen mittlern Fettwerth anzunehmen, beziehungsweise einen wie grossen Fehler man bei Annahme eines solchen Mittelwerthes machen kann. Vo i $t^{1}$ ) hat bei seinen Untersuchungen, die ihm als Grundlage für die wichtigsten Fragen der StoffwechselLehre dienen, einen solchen mittleren Werth von $0,91 \%$ angenommen. Da es wichtig ist, zu erfahren, wie Vo it zu diesem Werthe gelangt ist, führe ich seine Bemerkungen über diesen Punkt hier an. Er sagt ${ }^{2}$ ): "Bei einer von mir gemachten Analyse liessen sich aus bei $100^{\circ}$ getrocknetem reinem Fleisch $3,77 \%$, also aus frischem $0,91 \%$ Substanz mit Aether ausziehen." Es scheint also darnach nur e i n e Analyse gewesen zu sein, nach der $V o$ it seinen durchgebends für das gefütterte Fleisch bezüglich des Fettgehaltes gebrauchten Werth angenommen hat. Sechs Jahre später bemerkt

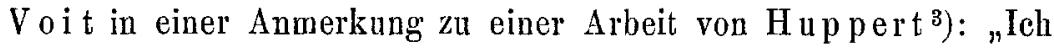
habe zu meinen Versuchen nicht beliebiges Muskelfleisch, sondern nur das Fleisch ungemïsteter ausgewachsener Rinder mit $0,9 \%$ Fett, geftittert." Dieselbe Angabe macht $\mathrm{V}_{0}$ i $\mathrm{t}$ auch in $\mathrm{Her-}$ m a n n's ${ }^{4}$ ) Handbuch, obschon inzwischeń bereits die Arbeit von Petersen sowie die von Leyder und Pyro erschienen waren, welche die Verschiedenheit des Fettgebalts in den verschiedenen Muskeln nachgewiesen hatten. Obwohl $\mathrm{V}$ o it schon gelegentlich der ersten Angabe der Zahl 0,91\% die Bemerkung macht: „Ich behaupte auch nicht, dass sich das Fleisch absolnt gleich bleibt, denn dies beweist sich ohne Analyse," so hat er doch thatsächlich diese Zahl seinen sämmtlichen Bilanzrechnungen zu Grunde gelegt, wohl offenbar in der Meinung, dass der Fettgehalt des magern gereinigten Fleisches nur unerheblichen Schwankungen unterworfen sei. Wenn nun Voit in seiner erwähnten Analyse 0,91\% Fett im Fleisch gefunden hat, so geht zunächst aus D orme ye r's Versuchen hervor, dass dieser Werth nur ein Theil des wirklich in

1) Voit, C., Ueber Zersetzungen der stickstoffhaltigen Stoffe im Thierkörper. Zeitschr. f. Biol. Bd. I. S. 95.

2) a. a. 0. Der Belag für diese Analyse findet sich bei Bischoff und Voit: Gesetze der Ernäbrung des Fleischfressers. 1860. S. 304.

3) Huppert, H., Ueber den Stickstoffgehalt des Fleisches. Zeitschr. f. Biol. Bd. VII. S. 360 .

4) Hermann, Handbuch der Physiologie. Bd. VI. 1. S. 249. 
dem betreffenden Fleisch enthaltenen Fettes ausdriickt, dass also die Zahl jedenfalls zu niedrig war. Denn dass V o it damals im Besitze einer Methode gewesen sei, die eine wirklich vollständige Erschöpfung des Fleischpulvers mit Aether ermöglicht, ist wohl nicht wabrscheinlich. Ferner aber war die Frage, ob nicht die thatsächlich vorkommenden Schwankungen im Fettgehalt viel grösser sind, als von $\mathrm{V}$ o it offenbar angenommen worden war.

Der Plan meiner Versuche ging also dahin, durch Vornabme einer grössern Anzahl von Fettbestimmungen in möglichst magerm Fleische iiber diese Punkte Aufklärung zu erbalten. Betreffs der Ausführung derselben habe ich mich im Wesentlichen Dormeyer angeschlossen, doch nicht wie er, die Substanz getrocknet, und zunäebst im Soxhlet'schen Apparat extrahirt, sondern gleich das feuchte Fleisch zur Verdauung gebracht.' Ausserdem habe ich einige kleinere Abänderungen getroffen, weshalb icb kurz auf die Methode hier zurïckkommen will.

Eine gewogene Menge Fleisch wurde in eine bestimmte Quantität Verdauungsflüssigkeit gebracht, und in den Brütofen gesetzt, wo eine Temperatur von circa $38-40^{\circ} \mathrm{C}$. unterhalten wurde. Nachdem das der Magenverdauung unterliegende Gewebe anfgelöst war, was verschieden lange Zeit in Anspruch nahm (1 bis 3 Tage), schwamm das Fett auf der Flitssigkeit und wurde beim Filtriren anf dem Filter gewonnen oder vorher im Aether anfgelöst und mit einer Pipette abgehoben. Letzteres Verfahren eignet sich besonders dann, wenn das Fleisch sehr fetthaltig ist, indem in diesem Falle das Filter leicht undurchlässig wird. Das Filtrat wurde in einem grossen Scheidetrichter mit etwa der gleiclien Menge Aether ansgeschüttelt, dann durch den Habn des Scheidetrichters die Flüssigkeit von dem Aether getrennt und der letztere verdunstet. Mit der Flüssigkeit wurde dasselbe Verfahren so lange wiederbolt, bis dieselbe mit Aether erschöpft war, was dann in der Regel eintrat, wenn 7 . bis 8 mal je eine halbe Stunde mit immer neuem Aether intensiv geschüttelt worden war. Bisweilen wurde die Trennung von Aether und Flïssigkeit dadurch erschwert, dass der Aether nach dem Schütteln eine schleimig aussehende emulsionsartige Truibung zeigte, die erst nach stundenlangem Stehen sich niedersenkte und in der unter dem Aether stehenden Flibssigkeit verschwand. Dieser Schwierigkeit liess'sich aber auf sehr einfache Weise dadurch begegnen, dass man den Scheidetrichter 
unter der Wasserleitung abkühlte, wodurch dann die sofortige Trennung mit scharfer Grenzfläche eintrat. Ja, es genügte auch schon, wenn man mit der Wand des Scheidetrichters den kalten Steinfussboden berührte, um denselben Erfolg zu erzielen. Das Filter, welches die Hauptmenge des Fettes enthielt, wurde getrocknet, in Stücke zerschnitten und diese in die Papierhülse des Soxhlet'schen Apparates gebracht und 3-4 Tage je 10 Stunden extrahirt. Der Vorgang wurde dann mit neuem Aether wiederholt, worauf bei der folgenden Extraction meist kein Fett mehr zu erhalten war. Den gewonnenen Extract behandelte ich in der Weise, dass derselbe zuerst auf $80^{\circ} \mathrm{C}$. erhitzt und dann wieder mit Aether aufgenommen wurde. Nachdem auch dieser verjagt war, wurde das Glas kurze Zeit bei $50^{\circ}$ C. gehalten, dann im Exsiccator über Schwefelsäure bis' zum Erkalten stehen gelassen und darauf gewogen.

Nachstehend noch einige Einzelheiten zu dem Verfahren.

Das untersuchte Fleisch stanmt sämmtlich von frisch geschlachteten, magern Kühen aus dem liesigen Schlachthause, wo es mir durch giitige Vermittelang des Hern Schlachthausverwalters Kreisthierarzt a. D. Brebeck möglich war, die gewünschten Qualitäten und Quantitäten völlig frisch zu erlangen. Da die obenerwähnten Untersuchungen von Peters en sowic die von Leyder und Pyro grosse Sehwankungen im Fettgehalt ergeben hatten, je nachdem das Material zur Untersuchung vom Vorder- oder Hintertheil genommen wurde, so benützte ich in Berïcksichtigung dieser Thatsache ebenfalls Muskeln, die theils der vordern, theils der hintern Hälfte der Thiere angehörten, nämlich den Pectoralis, Glutaeus und Rectus cruris, Muskeln, bei denen, da sie bei der Arbeit der Thiere vorzugsweise thätig sind, schon von vorne berein, ein relativ geringer Fettgehalt zu erwarten war. Das Fleisch wurde, in Zinnfolie verpackt, in das physiologische Institut gebracht und sogleich verarbeitet. Zunächst wurde das anssen anhaftende Fett genau entfernt, dann das Fleisch mit Messer und Scheere so klein zerschnitten wie möglich, und jedes sich dem Auge als Fett darbietende Theilchen wiederum auf das sorgfältigste entfernt. Um zu zeigen, wie peinlich ich dabei vorging, bemerke ich, dass sogar die sichtbaren Nervenbestandtheile herauspräparirt wurden und dass durchgängig $1 \frac{1}{2}$ Stunde nöthig waren, $80 \mathrm{gr}$ auf diese Weise gereinigtes Fleisch zu erbalten. Dasselbe wurde sodann gründlich 
durcheinander gemischt, zwischen* Uhrschalen vertheilt und gewogen. Selr gute Resultate lieferte auch das Abschaben des Fleisches mit dem Messer, ein Verfahren, das den Vorzug der grössern Schnelligkeit hat und bei etwas sorgfältiger Handhabung dieselben Ergebnisse liefert, wie ersteres, was C. Virchow ${ }^{\mathbf{1}}$ ) nachgewiesen hat.

Die Verdaungsflïssigkeit babe ich aus Schweinemagen in der Weise gewonnen, dass die gereinigte Oberfläche der Schleimhaut mit einem stumpfen Messer abgeschabt und der so erhaltene schleimige Saft in eine Salzsäurelösung von $0,5 \%$ gebracht wurde. Dieses Gemenge wurde einige Stunden bei circa $40^{\circ} \mathrm{C}$. digerirt, dann abgegossen und filtrirt. Späterbin präparirte ich vom Magen, nachdem der mit Galle imbibirte Pylorus-Theil abgeschnitten war, die Schleimhaut herunter, zerschnitt sie in kleine Stïcke und extrahirte kalt 3 bis 6 Stunden mit der Salzsäurelösung, goss ab und filtrirte wie vorher. Letztere Methode zog ich vor, weil bei ersterer das Filtriren durch den Schleim sebr erschwert wurde und längere Zeit in Anspruch nahm. Die so gewonnene Verdauungsflüssigkeit, von welcher zu den Analysen meistens je $100 \mathrm{ccm}$ verwandt wurden, war nun selbst nicht frei von Fett; es musste daher bei jeder Analyse besonders bestimmt werden, wieviel Fett in der. angewandten Verdauungsflïssigkeit schon vorher vorhanden war und diese Menge von der schliesslich erhaltenen in Abzug gebracht werden. Zur Bestimmung des in der Verdauungsflüssigkeit präformirten Fettes wurden in der Regel ebenfalls $100 \mathrm{ccm}$ derselben genommen, genau in der oben beschriebenen Weise mit Aether ausgeschittelt und das Fett in dem Aetherauszug bestimmt. Dabei wurden in $100 \mathrm{ccm}$ Flüssigkeit Fettmengen von 8 bis $13 \mathrm{mgr}$ gefunden.

Bei der Verdaung wurde, um dies hier zu erwähnen, das Fleisch nie vollständig aufgelöst, sondern es blieb in jedem Falle ein bald mehr körniger, bald mehr schleimiger Rückstand, über dessen Prozentgehalt ich, da sonst in der Litteratur dariiber nichts zu finden ist, einige Angaben mache.

Es blieben unverdaut von:

$$
\begin{aligned}
10,86 \text { gr Fleisch } 0,0842=0,77 \% . \\
10,48, \quad " \quad 0,090 !=0,86 \%
\end{aligned}
$$

1) R. Virchow's Archiv Bd. 84. S. 543. 


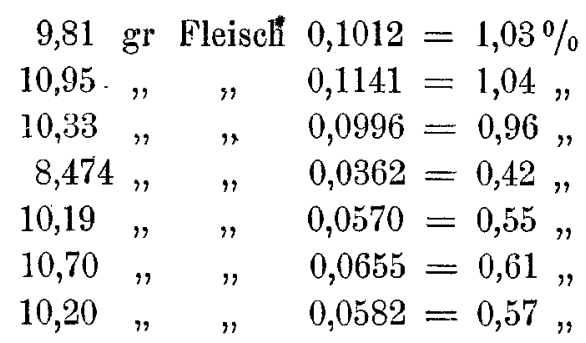

im Mittel also $0,76 \%$ oder auf trocken bezogen bei einem Trockengewicht von $25,82 \%=2,94 \%$.

Ich gebe nunmehr in Folgendem die Ergebnisse meiner Versuchsreihen, die sich alle, wie bereits oben gesagt, auf fenchtes Fleisch beziehen.

\section{Versuch I.}

Von zwei Kühen wird das Fleisch vom Musculus Pectus cruris, Glutaeus und Pectoralis genommen, sorgfältig vorbereitet und die gereinigte Substanz in je $100 \mathrm{ccm}$ Verdauungsflüssigkeit gebracht.

Es lieferte:

$\mathrm{Ku} \mathrm{h} \mathrm{I}$.

\begin{tabular}{|c|c|c|c|c|c|c|c|}
\hline $\mathbf{0} 1$ & $10,19 \mathrm{gr}$ & Fleisch & vom & Rectus & $=0,1049 \mathrm{gr}$ & Fett & $=1,02 \%$ \\
\hline$\Rightarrow$ & 10,48 & $"$ & $"$ & $n$ & $=0,1035 \%$ & $"$ & $=0,98$ \\
\hline$"$ & 10,70 & $"$ & " & Glutaeus & $=0,0893 \%$ & $"$ & $=0,83$ \\
\hline & 10,20 & 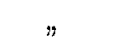 & 19 & 3 & $=0,0828$ & $\eta$ & $=0,81$ \\
\hline & 10,80 & 7 & $n$ & Pectoralis & $=0,1095$ & $"$ & $=1,00$ \\
\hline & 9,81 & $"$ & $"$ & $n$ & $=0,0964$ & " & $=0,98$ \\
\hline
\end{tabular}

$\mathrm{Ku} \mathrm{h}$ II.

Von $10,33 \mathrm{gr}$ Fleisch vom Rectus $=0,1230 \mathrm{gr}$ Fett $=1,19 \%$.

Kontrolprobe hiervon ging verloren.

Von 10,95 gr Fleisch vom Pectoralis $=0,3699$ gr Fett $=3,37 \%$. $" 8,48 " \quad " \quad=0,2769 " \quad "=3,30 \%$

In übersichtlicher Darstellung:

\begin{tabular}{c|c|c|c}
\hline \hline & M. Rectus & M. Glutaeus & M. Pectoralis \\
\hline \multirow{2}{*}{ Kub I } & $1,02 \%$ & $0,83 \%$ & $\mathbf{1 , 0 0 \%} \%$ \\
Kuh II & 0,98, & $0,81 "$ & $0,98 \%$ \\
" & $\mathbf{1 , 1 9 ,}$ & - & $3,37 \%$ \\
& - & - & $3,30 \%$
\end{tabular}




\section{Versiich II.}

Benützt wird das Fleisch von M. Pectoralis und M. Glutaens. Im Uebrigen wird wie bei Versuch I verfahren.

Es geben:

$10,15 \mathrm{gr}$ Fleisch von M. Pectoralis $0,0863 \mathrm{gr}$ Fett $0,85 \%$.

\begin{tabular}{|c|c|c|c|c|c|c|c|}
\hline 9,53, & $n$ & $"$ & $n$ & m & 0,0839 & $\eta$ & 0,88 \\
\hline 10,17, & $n$ & $n$ & M. & Glutaeus & 0,0842, & " & 0,82 \\
\hline 10,05 & $"$ & $"$ & " & " & 0,0812, & , & 0,80 \\
\hline
\end{tabular}

$\mathrm{Da}$ es mich interessirte zu erfahren, welchen Unterschied zwischen den Resultaten der von Salkowski empfohlenen und der von mir angewandten Methode besteht, beziehurgsweise wieviel Prozent des Gesammtwerthes nach ersterer erhalten werden kann, wurde der Versuch dahin erweitert, dass eine dritte Probe von M. Pectoralis genommen, zunächst bei $100^{\circ} \mathrm{C}$. getrocknet, vorsichtig gepulvert, dann in den S ox h let'schen Extraktor gebracht und 5 Stuuden ${ }^{1}$ ) mit warmen Aether behandelt wurde. Darauf wurde das. Fleischpulver, nachdem von demselben der Aether verjagt worden war, noch der Verdauung unterworfen.

Es lieferte:

\section{Die Extraktion:}

Von 9,41 gr Fleisch von Pectorales 0,511 gr Fett $=0,54 \%$.

$$
\text { Die Verdaung: }
$$

Derselben Fleischmenge 0,0341 gr Fett $=0,36 \%$.

Gesammtfett $=0,90 \%$. extracts.

Die Bestimmung nach Salkowski lieferte also $60 \%$ des Gesammt-

Uebersicht über Versuch II:

\begin{tabular}{c|c}
\hline M. Pectoralis & M. Glutaeus \\
\hline $0,88 \%$ & $0,82 \%$ \\
0,85, & $0,80 \%$ \\
0,90, & -
\end{tabular}

\section{Versuch III.}

Benutzt werden M. Pectoralis und M. Rectus. Verfahren wie bei Versuch I.

1) Salkowski verlangt bloss 3 Stunden. 
Es gaben:

$9,18 \mathrm{gr}$ Fleisch von Pectoralis $0,0905 \mathrm{gr}$ Fett $=0,98 \%$.

$9,20, ., \quad$ Rectus $0,0773, ",=0,84$,

$10,06, \quad " \quad, \quad 0,0825, . \quad, \quad=0,82$,

Auch hier werden, wie bei dem vorigen Versuche, zur Vergleichung mit den Ergebnissen der Salkowski'schen Methode 2 Proben, wie oben angegeben, behandelt.

Es geben:

a. 9,41 gr Fleisch von M. Pectoralis:

1. bei der Extraktion $0,0549 \mathrm{gr}$ Fett $=0,58 \%$.

2. bei nachträglicher Verdauung $0,0392 \mathrm{gr}$ Fett $=0,42 \%$

Gesammtfett 1,00\%. Die Bestimmung nach Salkowski lieferte also $58 \%$ des wirklichen Werthes.

b. $9,71 \mathrm{gr}$ Fleisch von M. Rectus:

1. bei der Extraction 0,0621 gr Fett $=0,63 \%$.

2. bei nachträglicher Verdauung $0,0362 \mathrm{gr}$ Fett $=0,37 \%$.

Gesammtfett $1,00 \%$. Nach S alk ow ski sind also nur $63 \%$ des ganzen Werthes erhalten.

Uebersicht über Versuch III.

\begin{tabular}{c|c}
\hline \hline M. Pectoralis & M. Rectus \\
\hline $0,98 \%$ & $0,82 \%$ \\
$1,00 "$ & $0,84 \%$ \\
- & $1,00 \%$
\end{tabular}

Die Ergebnisse der bisher berichteten Versuche beziehen sich alle auf äusserst sorgfältig von Fett befreites Fleisch. Um mir nun aber auch einen annähernden Begriff von dem Fettgehalt eines auch weniger peinlich ausgelesenen Stiickes Fleisch zu bilden, wie dies ja zu Futterzwecken bei Stoffwechselversuchen wohl nur in Betracht kommen kann, unternahm ich den folgenden

\section{Versueh IV.}

Benutzt wird das Fleisch von M. Glutaeus von 2 Kühen und nach dem Plane etwas gröber als sonst präparirt. Da es sehr schwierig ist, von dem Grade der Reinigung des Fleisches durch die Präparation eine sichere Vorstellung zu geben, ich aber missverständliche Deutungen vermeiden möchte, bemerke ich, dass der ganze Unterschied gegen früher darin bestand, dass das Fleisch in weniger kleine Stückchen zerlegt wurde. Die Auslese des Fettes geschah bei diesem Versuche mit derselben Sorgfalt, wie bei den frühern, und wurde circa $1 / 2$ Stunde gebraucht, um $60 \mathrm{gr}$ zu gewinnen. 
Die Resultate waren:

F ü r K n h I.

Von 8,17 gr Fleisch $0,2125 \mathrm{gr}$ Fett $=2,44 \%$.

, $10,00, " 0,2424, "=2,42$,

$\mathrm{F}$ ür K u h II.

Von 9,14 gr Fleisch 0,1796 gr Fett $=1,96 \%$ \%

" $9,77, \quad, \quad 0,1759,, \quad=1,80 "$

Uebersicht:

\begin{tabular}{c|c}
\hline \hline Kuh I & Kuh II \\
\hline $2,44 \%$ & $1,96 \%$ \\
2,42, & $1,80 \%$
\end{tabular}

Versuch V.

Endlich habe ich, um zu zeigen, einen wie grossen Einfluss das sorgfältige Auslesen des Fleisches hinsichtlich des Fettgehaltes ausübt, und zugleich die Schwankungen zu zeigen, denen die Ausbeute an Fett je nach der Präparation unterliegt, noch einen Versuch hier zugefügt, in welchem Kuhfleisch von M. Pectoralis benutat wurde, das nicht von einem ausgesucht magern Thier stammt, aber trotzdem dem Ansehen nach nur wenig Fett zu enthalten schien. Von diesem Fleische wurde eine Portion ohne weitere Vorbereitung in Arbeit genommen; die andere in der oben beschriebenen sehr sorgfältigen Weise von allem sichtbaren Fett befreit.

Es ergab sich :

a. Aus dem gereinigten Fleisch:

Von 10,04 gr Substanz 0,2350 gr Fett $=2,33 \%$.

" $10,06 " \quad 0,2240 " n=2,23$ "

b. Aus dem ungereinigten Fleisch:

Von 11,28 gr Substanz 1,0402 gr Fett $==9,22 \%$.

" $10,22 " \quad 0,9739, "=9,53 "$

Uebersicht.

\begin{tabular}{c|c}
\hline $\begin{array}{c}\text { Gereinigtes } \\
\text { Fleisch }\end{array}$ & $\begin{array}{c}\text { Ungereinigtes } \\
\text { Fleisch }\end{array}$ \\
\hline $2,33 \%$ & $9,22 \%$ \\
2,23, & $9,53 \%$
\end{tabular}


Es finden sich bei sämmtlichen Versuchen Verschiedenheiten grösserer oder kleinerer Art bei Fleisch derselben Muskeln beziiglich des Fettgehaltes. Dieselben erklären sich aber leicht einmal daraus, dass selbst beim exactesten Präpariren manchmal ein Theilchen Fett stehen bleibt, das bei der geringen Menge des Fettes gleich ins Gewicht fällt, sodann aber auch aus dem ungleichmässigen Fettgehalt der Muskeln. Will man diese Unterschiede in den Resultaten vermeiden, so darf man nicht mit feuchtem Fleisch arbeiten, sondern muss trocknen und pulverisiren, wobei eine fast homogene Substanz erzielt wird.

Ich hahe im Vorberigen stets Fett und Aetherextract als gleichwerthige Begriffe gebraucht und kann dies auch nach Dormeyer's Angaben. Aus demselben Grunde habe ich auch keine Untersuchungen über die Natur des Aetherextrakts gemacht, sondern verweise in dieser Beziehung auf Dorme yer's Arbeit.

Ich stelle nun in Folgendem zwecks besserer Uebersicht die einzelnen Werthe für den Fettgehalt hier zusammen.

\begin{tabular}{c|c|c|c}
\hline & M. Rectus & M. Glutaeus & M. Pectoralis \\
\hline Versuch I & & & \\
Kuh I & 1,02 & 0,83 & 1,00 \\
Kub II & 0,98 & 0,81 & 0,98 \\
Versuch II & 1,19 & - & 3,37 \\
& - & - & 3,30 \\
Versuch III & - & 0,82 & 0,88 \\
& 0,82 & 0,80 & 0,85 \\
Versuch IV 1) & 1,84 & - & 0,90 \\
Kuh I & - & - & 0,98 \\
& - & 2,44 & 1,00 \\
Kuh II & - & 2,42 & - \\
Versuch V $\left.{ }^{2}\right)$ & - & 1,96 & - \\
& - & - & - \\
& - & - & 2,80 \\
\hline
\end{tabular}

1) Betrifft das Fleisch, welches bei der Präparation weniger sorgfältig zerkleinert wurde.

2) Betrifft das weniger magere Fleisch. 
Aus vorstehender Tabelle ergibt sich zunächst, d a s s e s u nmöglicb ist, eine Mittelzabl festzustellen, da die einzelnen Werthe zu weit auseinander gehen und völlig abhängig sind von dem Grade der Präparation nnd den individuellen Schwankungen im Fettgehalt. Daber kann ich auch die Angabe Voit's, dass mageres Kuhfleiseh einen Fettgehalt von $0,91 \%$ besitze, weder bestätigen noch anch derselbeu eine andere Zahl gegenuiber stellen.

Es ist vielmehr nöthig, dass der Fettgehalt des Fleisches in jedem einzelnen Falledurcheine neue Analyse festgestellt wird. Dass der von Vo it gefundene Werth jedenfalls niedriger war, als der wirkliche Feltgehalt des von ihm untersuchten Fleisches, wurde bereits oben begründet. Wenn ich auch in einer Anzabl von Bestimmungen ähnliche Zablen erhalten habe, so wird dadurch die Richtigkeit der Voit'schen Werthe nicht etwa bestätigt, da mei n e Resultate der thatsächliche Ausdruck des im Fleische vorhandenen Fettes sind und nicht, wie bei ihm, nur einen Theilwerth des Ganzen darstellen. Neben dem Unterschiede in der Methode der Fettanalyse fällt zudem bei der Vergleichung meiner Werthe ond $V_{0}$ it's Zahlen noch ein weiterer Umstand ins Gewicht, das ist ive Art der Vorbereitung des Fleisches. Ein derartig sorgfältiges Auslesen und Reinigen des Fleisches, wie ich es in meinen Versuchen angewandt habe, würde bei Fütterungsversuchen, in welchen $1 \frac{1}{2}$ bis $21 / 2$ Kilo Fleisch zu verarbeiten sind, einfach andurehfuhrbar sein, da die Zubereitung einer Tagesportion nicht einige Stunden sondern Tage erfordern würde. Vo it gibt an, dass er mit seinem Diener manchmal 34 Stunden gebrancht habe, um 1500-2000 gr' fettfreies Fleisch zu gewinnen. Ich habe um $80 \mathrm{gr}$ zu erhalten, durebschnittlich $1 \frac{1}{2}$ Stunde gebraucht.

Ich fasse die Ergebnisse meiner Arbeit in folgenden Sätzen zusammen:

I. Ein Mittelwerth für den Fettgehalt im magern Fleische lässt sich nicht aufstellen.

II. Voit's Werth von $0,91 \%$ für den Fettgehalt im magern Kuhfleisch entspricht in keiner Weise den thatşächlichen Verhältnissen.

III. Mit Salkowski's Methode der Fettbestimmung im Fleische gelang es mir nur ca. $60 \%$ des Gesammitfettwerthes zu erhalten. 
358 Steil: Lässt sich etc. Fleisch von bestimmtem Nährwerth gewinnen?

IV. Die Angabe von Petersen, dass der Fettgehalt des Rindes im Hintertheil bedeutend grösser sei, als im Vordertheil, habe ich nicht bestätigt gefunden.

Am Schlusse der Arbeit ist es mir eine angenehme Aufgabe, Herrn Geheimrath $P$ fl ïg e r für die gütige Ueberlassung des Themas und die freundliche Unterstïtzung bei Anfertigung der Arbeit meinen herzlichsten Dank zu sagen. Desgleichen thue ich dies deu Herren Privatdocent Dr. M. Bleibtreu und Assistent Dr. Dormeyer für ihr bereitwilliges Entgegenkommen. 